\title{
CARACTERIZACIÓN FISICOQUÍMICA DE OLEOGELES DE ACEITE DE AGUACATE (Persea americana) Y SACHA INCHI (Plukenetia volubilis)
}

\section{PHYSICOCHEMICAL CHARACTERIZATION OF OLEOGELS OF AVOCADO OIL (Persea americana) AND SACHA INCHI (Plukenetia volubilis)}

\begin{abstract}
Merly E. Álvarez-H. ${ }^{1}$, Héctor J. Ciro-V. ${ }^{2}$, Julio C. Arango-T. ${ }^{3}$
${ }^{1}$ Esp y M.Sc., Ciencia y Tecnologia de Alimentos, Estudiante-Universidad Nacional de Colombia, Facultad de Ciencias Agrarias, Universidad Nacional. Medellín, Colombia. Dir: Cra 50 A \#57-51 Apto 504, cel:3002732497, e-mail: mealvareh@unal. edu.co; ${ }^{2}$ Ph.D., Ingeniería de Alimentos, Docente Universidad Nacional de Colombia, Sede Medellín. Facultad de Ciencias Agrarias, e-mail: hjciro@unal.edu.co; ${ }^{3}$ M.Sc., Docente Universidad Nacional de Colombia, Sede Medellín. Facultad de Ciencias Agrarias, e-mail: jcarango@unal.edu.co
\end{abstract}

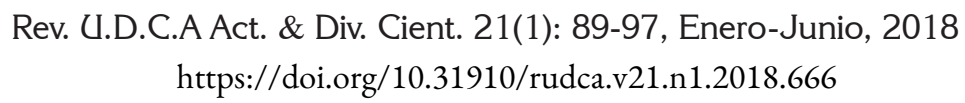

RESUMEN

Debido a las consecuencias que presenta en la salud una dieta poco saludable y con gran contenido de grasas trans, se han desarrollado alternativas alimentarias para el cuidado de la salud, entre ellos, los alimentos naturales con el mínimo aporte de carbohidratos y de grasas saturadas. Por lo anterior, se propuso la formulación de oleogeles libre de grasas trans, a partir de emulsiones (w/o), usando aceite de aguacate y sacha inchi, estructurados con diferentes emulsificantes. Se consideró un diseño factorial 3x3, donde se varió el tipo de emulsificante: lecitina, monoesterato de glicerilo y la relación entre el aceite de aguacate y el aceite de sacha inchi para la fase oleosa del sistema oleoestructurado. Se obtuvo un producto con tintes verdosos y amarillos, cuya cromaticidad b* en sistema Cielab es afectado por el tipo de emulsificante, tomando tonalidades más amarillas, cuando se usa la mezcla lecitina-Monoesterato de glicerilo. Las formulaciones, con una relación de aceites 70/30 usando Lecitina, como emulsificante y la formulación con una relación de aceites 80/20 y usando Monoesterato de glicerilo, mostraron los mejores resultados en términos de fuerza firmeza, en contraste con las margarinas comerciales, cumpliendo con los requisitos fisicoquímicos, establecido en las normas vigentes para aceites y margarinas, como son: índice de peróxido ( $<10 \mathrm{~m}$-eq $\mathrm{O}_{2} / \mathrm{kg}$ de grasa), acidez ( $<0,8 \%$ de ácido oleico) y contenido de humedad ( $\geq 16 \%$ b.h).

Palabras clave: Aceites insaturados, aceites vegetales, caracterización, firmeza.

\section{SUMMARY}

Due to the health consequences of an unhealthy diet with a high content of trans fats, food alternatives for health care have been developed, including natural foods with the minimum amount of carbohydrates and saturated fats. Therefore, the formulation of oleogeles free of trans fats was proposed from (w/o) emulsion using avocado and sacha inchi oil and different emulsifiers. A $3 \times 3$ factorial design was used in which the type of emulsifier: lecithin, glyceryl monostearate and the ratio of avocado and sacha inchi oil was varied. A product was obtained with greenish and yellow dyes, whose chromaticity $b^{*}$ is affected by the type of emulsifier, taking more yellow tones when the lecithin-glyceryl monostearate mixture is used. Formulations with a 70/30 ratio of oils using Lecithin as an emulsifier agent and formulation with an $80 / 20$ oil ratio and using glyceryl monostearate showed the best results in terms of firmness in contrast to commercial margarines, meeting the physico-chemical requirements established in the current standards for oils and margarines such as: peroxide value $\left(<10 \mathrm{~m}\right.$-eq $\mathrm{O}_{2} / \mathrm{kg}$ fat), acidity $(<0.8 \%$ oleic acid) and moisture content ( $\geq 16 \%$ w.b).

Key words: Unsaturated fats, plant oils, characterization, firmness

\section{INTRODUCCIÓN}

Factores, como el sedentarismo y el consumo de alimentos poco saludables, han hecho que aumenten las cifras de la población que padece de las denominadas enfermedades no trasmisibles (ENT), en las que se destaca la obesidad, la 
diabetes Mellitus, los problemas cardiovasculares y la hipertensión (OPS/OMS, 2016). El riesgo de enfermedades crónicas ha ido creciendo, a medida que las pautas alimentarias de la población cambiaban y se incorporaban cada vez más alimentos procesados ricos en grasas, azúcares o con alto contenido de ácidos grasos trans (AGT), de producción industrial (Bejarano-Roncancio et al. 2015)

Recientes estudios han comprobado que el consumo de alimentos ricos en grasas trans, sal y carbohidratos favorecen el incremento de peso y de los factores de riesgo de contraer alguna enfermedad, del tipo ENT (Parodi, 2009).

El aceite de aguacate (Persea americana) es rico en ácidos grasos mono-insaturados, además posee tocoferoles de gran beneficio al ser consumidos; es fuente importante de vitaminas, contiene 12 de las 13 vitaminas existentes, (Barbosa-Martín et al. 2016). Por su parte, el aceite de Sacha inchi, obtenido de las semillas de esta planta oleaginosa (Plukenetia volubilis L.), se caracteriza por alto porcentaje de ácidos grasos poli-insaturados, principalmente, linolénico y linoléico, los cuales, representan el $82 \%$ del contenido total de aceite (Fanali et al. 2011).

Las propiedades fisicoquímicas de las moléculas tensoactivas son de gran interés, porque determinan la estabilidad de las emulsiones. Los fosfolípidos, como la Lecitina (LC) y los monoglicéridos, como el Monoesterato de glicerilo (ME), son ejemplos comunes de emulsificantes de bajo peso molecular, utilizados en la estabilización de diferentes productos alimenticios, ya que son compuestos muy activos en la superficie (Moran-Valero et al. 2017).

La LC y el ME son emulsionantes que se necesitan para aumentar la estabilidad coloidal y proporcionar interacciones interfaciales entre los componentes alimentarios, que son factores importantes para mejorar aún más la estabilidad de la emulsión y la vida útil en muchos alimentos. Estas aplicaciones, incluyen formulaciones de alimentos tradicionales, como productos de panadería, confitería o productos cárnicos, entre otras o nuevas formulaciones, como los alimentos instantáneos y bajos en grasa, formulaciones alimenticias de alto o bajo contenido en alcohol, alimentos funcionales (Moran-Valero et al. 2017).

El objetivo del presente trabajo fue estudiar el efecto de la adición del tipo de emulsificante y la relación de aceite sobre las propiedades fisicoquímicas de emulsiones estructuradas, a partir de aceite de aguacate y sacha inchi, para la estructuración de un oleogel, como sustituto de grasas usadas en mesa y en cocina, libre de grasas trans.

\section{MATERIALES Y MÉTODOS}

El aceite de aguacate ( $P$. americana) fue suministrado por Terravocado S.A., el aceite de sacha inchi ( $P$. volubilis), por un productor local de la Amazonia colombiana y la grasa alimentaria de origen vegetal (shortening latino), de Tecnas S.A. Además, se utilizaron emulsificantes: Lecitina (LC) y monoesterato de glicerilo (ME) y conservantes de calidad alimentaria, como ácido cítrico, sal y Terbutil Hidroquinona (TBQH), estos últimos de Bell Chem Internacional S.A. (Medellín, Colombia).

Formulación de las emulsiones: En las preparaciones de las emulsiones (w/o), se inició con la fase lipídica: aceite de aguacate/aceite de sacha inchi, shortening latino y TBQH y, posteriormente, la fase acuosa. En esta fase, se le adicionó los conservantes, como el cloruro de sodio y el ácido cítrico, ingredientes con la particularidad de ser solubles en agua. Se homogenizó térmicamente, a temperatura de $50^{\circ} \mathrm{C}$, para favorecer la mezcla. La proporción de cada uno de los componentes del sistema oleoestructurado se midió en peso, para evitar errores, debidos a la variación de volumen por la temperatura.

En este estudio, se estableció una relación (w/o) de 20/80, donde un $30 \%$ de la fase oleosa en todas las muestras corresponde a la grasa de origen vegetal (shortening latino). Adicionalmente, la concentración de los aditivos y de los conservantes, como ácido cítrico y cloruro de sodio, fue establecido en $1 \%$ p/p. La homogenización de las dos fases, se realizó mediante el agitador Ultaturrax T25y, proceso, en el cual, previamente, se realizaron ensayos para determinar los valores óptimos de las variables, que se dejaron fijas en la preparación de las emulsiones: temperatura de homogenización $\left(50^{\circ} \mathrm{C}\right)$, la velocidad de agitación (4000rpm) y tiempo (10 minutos).

Estructuración sólida del oleogel: Para favorecer el proceso de cristalización y formar los oleogeles, las emulsiones estructuradas fueron sometidas a un proceso de enfriamiento durante $12 \mathrm{~h}$, a una temperatura de $4^{\circ} \mathrm{C} \pm 1$. Una vez obtenido el sistema solidificado, las muestras continuaron en refrigeración, a esta temperatura.

Diseño experimental: Se estableció un diseño factorial 3x3 completamente aleatorizado, para un total de 9 tratamientos. Los factores y los niveles establecidos en el estudio, se encuentran en la tabla 1 . Se analizaron los datos experimentales estadísticamente, con un nivel de significancia del $5 \%$, por medio de análisis de varianza (ANAVA), pruebas de diferenciación de medias, usando Statgraphics Centurion XVII software (versión 17.1.12, USA). 
Tabla 1. Diseño experimental para la preparación de emulsiones.

\begin{tabular}{|c|c|c|}
\hline & \multicolumn{2}{|c|}{ Factores } \\
\hline Emulsión & $\begin{array}{c}\text { Relación de aceites aguacate/sacha } \\
\text { inchi }\end{array}$ & Tipo de emulsificante \\
\hline E1 & $70 / 30$ & ME \\
\hline E2 & $90 / 10$ & $\mathrm{LC} / \mathrm{ME}$ \\
\hline E3 & $70 / 30$ & $\mathrm{LC} / \mathrm{ME}$ \\
\hline E4 & $70 / 30$ & $\mathrm{LC}$ \\
\hline E5 & $80 / 20$ & $\mathrm{LC} / \mathrm{ME}$ \\
\hline E6 & $90 / 10$ & $\mathrm{LC}$ \\
\hline E7 & $90 / 10$ & ME \\
\hline E8 & $80 / 20$ & ME \\
\hline E9 & $80 / 20$ & $\mathrm{LC}$ \\
\hline
\end{tabular}

Determinación del color: El aspecto visual de los alimentos es una serie de características físicas, que incluyen el color, la turbiedad, la transparencia, entre otros y que, en conjunto muestran un panorama de la calidad del producto, razón por la cual, el color se puede usar como indicador de las transformaciones naturales o de los cambios ocurridos en el proceso de preparación de la emulsión. El color es definido como un haz de radiaciones luminosas, con una distribución espectral; los alimentos transparentes transforman la luz que les llega y la transmiten, a través de sus cuerpos, mientras que, los alimentos opacos, la reflejan. Esta transformación de la luz emitida incide en la retina del ojo y es la que, finalmente, conlleva a la evaluación final del producto. La evaluación colorimétrica, se realizó a través el sistema de coordenadas CIELAB, dado por un sistema cromático, mediante las coordenadas L, a* y b* (Moyano et al. 2008). Para este propósito, se utilizó el colorímetro X-RITE, modelo SP60, iluminante D65 y ángulo visual de $10^{\circ}$. A partir de los espectros, se obtuvo las coordenadas de color del CIEL*a*b*, donde $\mathrm{L}^{*}$ es un indicador de la luminosidad $\mathrm{a} *$ (cromaticidad verde $[-]$ a rojo [+]) y b* (cromaticidad azul [-] a amarillo [+]).

Firmeza: La medición de la fuerza máxima de penetración en los oleogeles, se llevó a cabo en el analizador de textura TA-XT2i. El espesor de la muestra fue de $4 \mathrm{~cm}$; la sonda del equipo utilizada fue cónica, con ángulo de penetración de $30^{\circ}$ (P/30C); la celda de carga fue 50kgf; la velocidad de deformación de $1 \mathrm{~mm} / \mathrm{s}$ y máxima profundidad de penetración de $1 \mathrm{~cm}$. La fuerza de firmeza fue considerada como la máxima de la curva de flujo del material (fuerza vs deformación). Se realizaron tres réplicas, donde la muestras a analizar fue el sistema oleo-estructurado solidificado durante refrigeración, a $4^{\circ} \mathrm{C} \pm 1$.
Determinación del índice de acidez (IA): El contenido de ácidos grasos libres, se determinó mediante la norma AOCS Official Method Ca, de 1997, expresado en porcentaje de ácido oleico.

Determinación del contenido de humedad: Para determinar la humedad en las muestras, se pesaron $3,00 \pm 0,01 \mathrm{~g}$, que fueron sometidos a una temperatura de $105^{\circ} \mathrm{C}$, en un horno de convección forzada, durante $24 \mathrm{~h}$, hasta alcanzar un peso constante. La humedad, se determinó por diferencia de peso, de acuerdo con la norma AOCS Official Method 930.15, de 1990.

Determinación del índice de peróxidos: El análisis, se llevó a cabo mediante la norma AOCS Official Method cd 8-53, de 2003. Este método permite cuantificar la presencia peróxidos provenientes de la oxidación primaria de los lípidos. Estos tienen la capacidad de oxidar los iones hierro $\left(\mathrm{Fe}^{+2} \mathrm{a}\right.$ $\mathrm{Fe}^{+3}$ ), en soluciones de bajo $\mathrm{pH}$. La oxidación resultante se cuantifica con ayuda de tiocianato que acompleja los iones hierro $\left(\mathrm{Fe}^{+3}\right)$, generando una coloración medible por espectrofotometría UV.

\section{RESULTADOS Y DISCUSIÓN}

Color: La prueba ANAVA mostró diferencias significativas ( $\mathrm{p}$ $<0,05$ ), para el valor de la cromaticidad $b^{*}$ con respecto al tipo de emulsificante, donde los valores variaron entre 19,11 y 26,41 , mientras que para los valores de la luminosidad $L * y$ la cromaticidad $a *$ no se presentaron diferencias significativas para ninguno de los factores ni su interacción ( $p>0,05)$.

En la tabla 2, se muestras los valores de colorimetría, mediante el método CIELAB, para todas las formulaciones. Los 
valores a* negativos (coordenadas de verde a rojo), para las emulsiones, indican un ligero tinte verdoso, donde en la figura $1 \mathrm{E}$, se puede observar que se obtienen valores más negativos, más tendientes al verde, cuando se usa una relación de aceite 90/10 y ME, como emulsificante, acorde con lo reportado para aceite de Sacha inchi, con valores de a* de -0,42 (Paucar-Menacho et al. 2015); para la oleína de palma, los valores de a* son alrededor de -6,86 (Vives, 2004) y los valores para la pulpa de aguacate variaba alrededor de -6,33 (Bi et al. 2015). El valor positivo de los valores de b * (coor- denadas de azul a amarillo) indican que todas las emulsiones tenían un tinte amarillento, que aumentaba cuando se usaba la mezcla de LC/ME y una relación de aceite 90/10, como se muestra en las interacciones (Figura 1F). Estas diferencias en el color de la emulsión pueden ser atribuidas a diferencias en los colores intrínsecos de los tensioactivos, ya que, tanto ME como la LC, tenía un color amarillento. Estas diferencias concuerdan con lo reportado en emulsiones de café, donde se usó lecitina (Chung et al. 2017).

Tabla 2. Valores de colorimetría CIELAB para las emulsiones usadas en la preparación del oleogel.

\begin{tabular}{|c|c|c|c|}
\hline \multirow{2}{*}{ Emulsión } & \multicolumn{3}{|c|}{ Parámetro de color } \\
\cline { 2 - 4 } & $\mathbf{L}^{*}$ & $\mathbf{a}^{*}$ & $\mathbf{b}^{*}$ \\
\hline E1 & $63,79 \pm 0,81$ & $-3,14 \pm 0,02$ & $20,82 \pm 0,18$ \\
\hline E2 & $64,40 \pm 0,48$ & $-3,08 \pm 0,24$ & $22,10 \pm 0,21$ \\
\hline E3 & $67,87 \pm 0,12$ & $-3,13 \pm 0,10$ & $20,85 \pm 0,13$ \\
\hline E4 & $64,18 \pm 0,25$ & $-3,22 \pm 0,19$ & $21,62 \pm 0,23$ \\
\hline E5 & $63,99 \pm 0,12$ & $-3,24 \pm 0,00$ & $22,11 \pm 0,16$ \\
\hline E6 & $63,14 \pm 0,56$ & $-3,32 \pm 0,11$ & $21,86 \pm 0,14$ \\
\hline E7 & $65,52 \pm 0,68$ & $-3,20 \pm 0,11$ & $21,85 \pm 0,13$ \\
\hline E8 & $62,54 \pm 0,16$ & $-3,08 \pm 0,01$ & $24,71 \pm 0,72$ \\
\hline E9 & $60,58 \pm 0,24$ & $-3,40 \pm 0,18$ & $21,36 \pm 0,81$ \\
\hline
\end{tabular}

En cuanto a la luminosidad L*, sus valores alrededor de 63, muestran que las emulsiones son claras, mientras que en el reporte de aceite de aguacate, los valores fueron 11,52 (Castañeda-Antonio et al. 2015) y de 77,73 fueron obtenidos para aceite de Sacha inchi (Paucar-Menacho et al. 2015).De igual forma, el shortening latino está compuesto, principalmente, por oleína y por estearina de palma, cuyo valor de L* oscila alrededor de 80,72 (Rincón \& Martínez, 2009), por lo tanto, la luminosidad de las emulsiones se puede atribuir, especialmente, al aceite de sacha inchi y al shortening latino.

\section{Propiedades texturales de los oleogeles obtenidos: Esta} evaluación, se hizo por penetrometría cónica, cuya sonda de medición es conducida en el oleogel cristalizado y la fuerza requerida para determinada profundidad de penetración total fue medida. Esta técnica ha sido ampliamente utilizada en la caracterización textural en productos alimenticios (Zúñiga et al. 2007).

Los valores de fuerza máxima de penetración para los oleogeles obtenidos oscilaron entre 0,89 y $4,79 \mathrm{~N}$, valores comparables con los obtenidos de aceite de girasol oleoestruc- turados con monoglicéridos y fitoesteroles, donde la fuerza máxima varió entre 1,3 y 6,0N (Kouzounis et al. 2017). De igual forma, se obtuvo oleogeles con aceite de girasol estructurado con monoglicéridos, cuya fuerza máxima varió de 0,40 y 2,95N (Palla et al. 2017). En el mismo estudio, se comparó con una margarina comercial, cuya media para la fuerza máxima de penetración fue de 1,60N, por lo tanto, es posible el desarrollo de un material con firmeza similar a las margarinas comerciales.

Al evaluar el efecto de la fuerza máxima, la prueba ANAVA, con un 5\% de nivel de significancia, mostró que la interacción entre los factores tipo de emulsificantes y relación de aceites influye sobre la firmeza de los oleogeles y es estadísticamente significativa $(p<0,05)$. Por diferencia de medias, se obtiene que el valor de la mayor fuerza de firmeza fue para la formulación del oleogel preparado con una relación de aceites 80/20 y usando LC, como emulsificante (Figura 1C). Este resultado difiere de los resultados obtenidos para queso estructurado con almidón de tapioca y lecitina, donde se evidenció disminución de la firmeza, cuando se usó lecitina, por el debilitamiento de las estructuras creadas por las proteínas, a través de la hidratación, pero hubo una acción sinérgica 

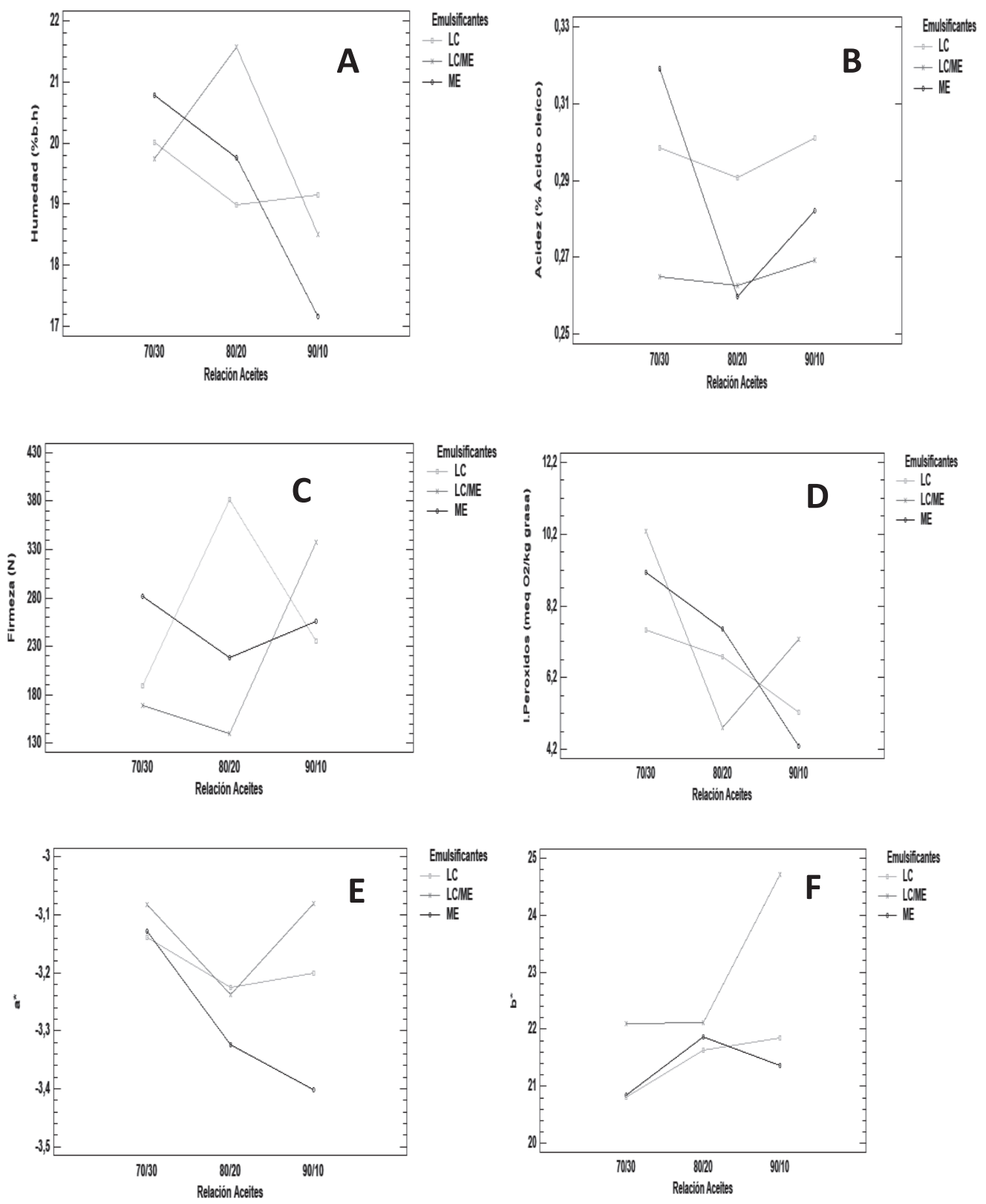

Figura 1. Interacciones de efectos para el tratamiento factorial: (A) Contenido de Humedad (\%b.h); (B) Acidez (\% ácido oleico); (C) Fuerza de Firmeza (N); (D) Índice de Peróxidos (meq O2/kg de grasa); (E) Valor de la coordenada CIELAB a*; (F) Valor de la coordenada CIELAB b*.

que mejoraba la firmeza, cuando se usaba la combinación almidón de tapioca y lecitina. De igual forma, cuando se evalúa lecitina y triestearato de sorbitán, los dos componentes, individualmente en aceites vegetales, no dan estructura a concentraciones entre 6 y $20 \%$ p/p, mientras que se observa un efecto sinérgico con su mezcla, en relaciones específicas de lecitina: triesterato de sorbitan, entre 40:60 y 60:40, obteniéndose geles firmes (Pernetti et al. 2007); sin embargo, en estudios de caracterización de hidrogeles de emulsión oleo-estructurado a base de lecitina de soja con aceite de sésamo, se formaron geles en emulsión, cuando la concentración de lecitina de soja era mayor al 40\% p/p (Singh et al. 2016); así mismo, se obtuvo sistemas oleoestructurados con aceite de girasol de dureza comparable con margarinas comerciales, cuando se usó $12 \%$ p/p, de monoglicéridos (Palla et al. 2017). 
Índice de acidez: Con el índice de acidez, se determina la presencia natural de la acidez libre en las grasas, resultado de la hidrólisis o la descomposición lipolítica de algunos triglicéridos (Hidrólisis enzimática, tratamiento químico, o acción bacteriana). El índice de acidez, se define como el número de miligramos de $\mathrm{KOH}$ que se requieren para neutralizar los ácidos grasos libres, contenidos en un gramo de grasa. Debido a que la acidez de las sustancias grasas es muy variable, generalmente, las grasas frescas o recién preparadas no contienen ácidos grasos libres o, si los contienen, los tienen en muy pequeñas cantidades; al envejecer, principalmente, si no han estado protegidos de la acción del aire y la luz, su acidez crece lentamente, al principio y con cierta rapidez, después (Baldo et al. 2016).

El análisis estadísticos para la acidez determinó que ninguno de los factores ni su interacción influyen sobre el índice de acidez, debido, posiblemente, a que en las formulaciones no varían en el contenido de grasa total sino la proporción entre los aceites de aguacate y de sacha inchi y, en ambos, predominan los ácidos grasos oleico y linoleico (Perez-Monterroza et al. 2014), por lo tanto, el contenido de los ácidos grasos libre no se ve modificado significativamente; lo anterior se observa en la Figura 1B. En la tabla 3, se muestra el valor de la acidez para todas las emulsiones, cuyo valores fueron iguales o inferiores a $0,3 \%$, que es el máximo permitido, según lo indicado en la Norma Técnica Colombiana NTC 241, de 2002, para margarinas e, inferiores al 0,8 y $1 \%$, que es el máximo permitido para aceite extra virgen de aguacate, según la Norma Técnica Colombiana NTC 199, de 2009 y para aceite de sacha inchi, según la Norma Técnica Peruana 151.400 , de 2009, respectivamente. Valores de $0,16 \%$ fueron reportados para el aceite de aguacate, obtenido por fluidos súper críticos y de $0,48 \%$, por métodos convencionales (Corzzini et al. 2017). Asimismo, se han reportado valores para aceite de sacha inchi, de 0,03 y 0,11\%, mediante extracción soxhlet y fluidos súper críticos, respectivamente (Castaño T. et al. 2012) y de $1,14 \%$, para aceite comercial (Paucar-Menacho et al. 2015). Valores en el índice de acidez se puede ver afectado por el tratamiento post-cosecha de la frutas o semillas, del método de extracción del aceite, exposición a la luz y a las altas temperaturas; de ahí, su variación en los valores reportados (Borges et al. 2017).

Contenido de humedad del sistema oleoestructurado: Para el contenido de humedad de las diferentes formulaciones, no se encontraron diferencias significativas $(P>0,05)$ para los factores relación de aceite y tipo de emulsificante. El contenido de humedad varió entre 17,17 y 21,56\% b.h, cumpliendo con el requisito de contenido de agua para los esparcibles de uso en mesa y en cocina. La norma colombiana NTC 241, de 2002, establece un porcentaje de humedad igual o superior al $16 \%$ b.h y en la figura $1 \mathrm{~A}$, se muestra las interacciones entre los factores.
Resultados similares en el contenido de humedad de 17,11 $\pm 0,41 \%$ b.h y $20,95 \pm 0,49 \%$ b.h, se obtuvieron en margarinas preparadas con extracto hidro-etanólico de cáscaras de nopal (Opuntia ficus-indica) y vitaminas E, respectivamente (Chougui et al. 2015).

Índice de peróxidos: El índice de peróxido determina el contenido de peróxido de hidrógeno y proporciona una medida de la oxidación de los ácidos grasos insaturados. En la tabla 3 , se observan los valores de índice de peróxido para todas las emulsiones, a excepción de una formulación, todos los valores se encuentran por debajo de la norma Mexicana, para aceite de aguacate, NMX-F-052-SCFI, de 2008 y de la Norma Técnica Peruana, para aceite de sacha inchi, de 2009, de $10 \mathrm{meq} \mathrm{O}_{2} / \mathrm{kg}$ de aceite.

Se han reportado valores para el índice de peróxido en aceite de sacha inchi de $7,5 \mathrm{meq} \mathrm{O}_{2} / \mathrm{kg}$ de aceite fresco (Vicente et al. 2015), mientras que para el aceite de aguacate se han reportado 5,26meq $\mathrm{O}_{2} / \mathrm{kg}$ de aceite fresco (Castañeda-Antonio et al. 2015); sin embargo, el valor del peróxido, se puede atribuir a muchos factores, como la variedad, las prácticas agrícolas y de las enzimas liberadas de las células de la fruta durante el proceso (Vargas-Ortiz et al. 2017).

Los resultados estadísticos mostraron efectos estadísticamente significativos $(p<0,05)$ para la relación de aceites, mientras que, el tipo de emulsificante no afecta el índice de peróxidos. Por diferencia de medias, se obtiene que una mayor proporción de aceite de sacha inchi favorece el proceso de oxidación o permite obtener valores más altos para el índice de peróxidos, como se observa en la figura 1D. En estudios realizados con aceites de ricino, de palma y de sacha inchi, se determinó que el aceite de ricino es el de menor grado de oxidación, lo que se explica por su bajo contenido ácidos grasos poliinsaturados, como linoleico y linolénico. Por el contrario, el aceite de sacha inchi es el de mayor grado de oxidación, debido a su alto contenido de ácidos linoleico y linolénico (Zuleta Suárez et al. 2012).

Es posible que el tipo de emulsificante no sea significativo en el índice de peróxido, debido a que, tanto la LC como el $\mathrm{ME}$, actúan como conservantes y como modificadores de componente reológico; por su parte, la LC cumple función antioxidante, su actividad se debe principalmente a la acción de tocoferoles; la lecitina purificada puede ser menos activa como antioxidante primario, pero puede actuar como sinergista, en virtud de la capacidad de ligar iones metálicos. El monoestearato de glicerilo, por su parte, también es utilizado como agente conservador, debido a que actúa encapsulando las gotas de aceites presentes en la emulsión (Wang \& Marangoni, 2016). 
Tabla 3. Caracterización físicoquímica de las emulsiones.

\begin{tabular}{|c|c|c|c|c|}
\hline EMULSIÓN & $\begin{array}{c}\text { Peróxidos } \\
\text { (meq O } / \text { /kg de grasa) }\end{array}$ & $\begin{array}{c}\text { Acidez } \\
\text { (\% ácido oleico) }\end{array}$ & $\begin{array}{c}\text { Humedad } \\
\text { (\%, b.h) }\end{array}$ & Firmeza (N) \\
\hline E1 & $9,14 \pm 0,43$ & $0,30 \pm 0,00$ & $20,79 \pm 0,27$ & $2,81 \pm 0,03$ \\
\hline E2 & $7,28 \pm 0,21$ & $0,27 \pm 0,07$ & $18,50 \pm 0,31$ & $3,36 \pm 0,05$ \\
\hline E3 & $10,29 \pm 0,15$ & $0,27 \pm 0,07$ & $19,75 \pm 0,11$ & $1,68 \pm 0,02$ \\
\hline E4 & $7,52 \pm 0,31$ & $0,30 \pm 0,04$ & $20,00 \pm 0,16$ & $1,89 \pm 0,09$ \\
\hline E5 & $4,8 \pm 0,19$ & $0,26 \pm 0,00$ & $21,56 \pm 0,17$ & $1,40 \pm 0,05$ \\
\hline E6 & $5,25 \pm 0,01$ & $0,30 \pm 0,06$ & $19,15 \pm 0,03$ & $2,35 \pm 0,02$ \\
\hline E7 & $4,30 \pm 0,10$ & $0,28 \pm 0,04$ & $17,17 \pm 0,04$ & $2,56 \pm 0,01$ \\
\hline E8 & $7,55 \pm 0,46$ & $0,26 \pm 0,08$ & $19,76 \pm 0,05$ & $2,18 \pm 0,03$ \\
\hline E9 & $6,77 \pm 0,12$ & $0,29 \pm 0,03$ & $18,99 \pm 0,69$ & $3,81 \pm 0,03$ \\
\hline
\end{tabular}

En conclusión, se obtienen oleogeles con valores de acidez y humedad, según lo establecido en la normatividad vigente colombiana. Adicionalmente, valores de peróxidos más altos son alcanzados cuando se usa mayor proporción de aceite de sacha inchi, debido al gran contenido de ácidos grasos poliinsaturados; a excepción del oleogel estructurado, con una relación de aceites 70/30 y LC como emulsificante, todos cumplen con el máximo permitido, para el índice de peróxido, de acuerdo con las normas reguladoras.

Se desarrollaron oleogeles con fuerza de firmeza comparable con margarinas comerciales para las emulsiones preparadas con una relación de aceites $80 / 20$, usando como emulsificante Monoestertato de glicerilo o la mezcla lecitina -Monoesterato de glicerilo y la formulación con una relación de aceites 70/30, usando lecitina; sin embargo, dado que la emulsión preparada con una relación de aceite 70/30 y usando mezcla lecitina-Monoesterato de glicerilo-, no cumple en términos de índice de peróxidos, no se podría tomar como la mejor.

El tipo de emulsificante influyó en el color, específicamente, en la cromaticidad b*, que va del azul al amarillo, obteniéndose el valor máximo de cromaticidad, cuando se usa como emulsificante la mezcla LC/ME.

Agradecimientos: Facultad de Ciencias Agrarias, de la Universidad Nacional de Colombia. Conflictos de intereses: El manuscrito fue preparado y revisado con la participación de todos los autores, quienes declaramos que no existe conflicto de intereses que ponga en riesgo la validez de los resultados presentados.

\section{BIBLIOGRAFÍA}

1. AOCS Ca 5a-40. 1997. Official Method Free Fatty Acids, Sampling and Analysis of Commercial Fats and Oils. Copyright The American Oils Chemist's Society. (USA).

2. AOCS 930.15 1990. Official Method for the determination of moisture content. Copyright The American Oils Chemist's Society. (USA).

3. AOCS Cd 853. 2003. Official Method Peroxide Value Acetic Acid (Chloroform Method), And, Sampling and Analysis of Commercial Fats and Oils. Copyright The American Oils Chemist's Society. (USA).

4. BALDO, M.A.; OLIVERI, P.; SIMONETTI, R.; DANIELE, S. 2016. A novel electroanalytical approach based on the use of a room temperature ionic liquid for the determination of olive oil acidity Crossmark. Talanta. 161:881-887.

5. BARBOSA-MARTÍN, E.; CHEL-GUERRERO, L.; GONZÁLEZ-MONDRAGÓN, E.; BETANCUR-ANCONA, D. 2016. Chemical and technological properties of avocado (Persea americana Mill.) seed fibrous residues. Food and Bioproducts Processing. Part A.100:457463.

6. BEJARANO-RONCANCIO, J.; GAMBOA-DELGADO, E.M.; AYA-BAQUERO, D.H.; PARRA, D. 2015. Los alimentos y bebidas ultra-procesados que ingresan a Colombia por el tratado de libre comercio cinfluirán en el peso de los colombianos?. Chil. Nutr. 42(4):409-413. 
7. BI, X.; HEMAR, Y.; BALABAN, M.O.; LIAO, X. 2015 . The effect of ultrasound on particle size, color, viscosity and polyphenol oxidase activity of diluted avocado puree. Ultrasonics - Sonochemistry. 27:567-575.

8. BORGES, T.H.; PEREIRA, J.A.; CABRERA-VIQUE, C.; LARA, L.; OLIVEIRA, A.F.; SEIQUER, I. 2017. Characterization of Arbequina virgin olive oils produced in different regions of Brazil and Spain : Physicochemical properties. oxidative stability and fatty acid profile. Food Chemistry. 215:454-462.

9. CASTAÑEDA-ANTONIO, D.; LÓPEZ-VARELA, P.; GUEL-SILVA, G.; RAMOS-CASELLIS, E.; ARIZA-ORTEGA, A.; CARRERA-MARTÍNEZ, C.D.; PORTILLO-REYES, R. 2015. Caracterización oxidativa de aceite de aguacate hass y aceites de aguacate criollo (P. americana Mill . Var . Drymifolia ). Postcosecha e industrialización: VIII Congreso Mundial de la Palta. p.423-430.

10. CASTAÑO T., D.L.; VALENCIA G., M.DEL P.; MURILLO P., E.; MENDEZ A., J.J.; ERAS JOLI, J. 2012. Composición de ácidos grasos de sacha inchi (Plukenetia volubilis Linneo) y su relación con la bioactividad del vegetal. Rev. Chil.Nutr. 39(1):45-52.

11. CHOUGUI, N.; DJERROUD, N.; NARAOUI, F.; HADJAL, S.; ALIANE, K.; ZEROUAL, B.; LARBAT, R. 2015. Physicochemical properties and storage stability of margarine containing Opuntia ficus-indica peel extract as antioxidant. Food Chemistry.173:382-390.

12. CHUNG, C.; SHER, A.; ROUSSET, P.; ANDREW, E.; JULIAN, D. 2017. Formulation of food emulsions using natural emulsi fi ers : Utilization of quillaja saponin and soy lecithin to fabricate liquid coffee whiteners. J. Food Engineering. 209:1-11.

13. COMISIÓN DE NORMALIZACIÓN Y FISCALIZACIÓN DE BARRERAS COMERCIALES NO ARANCELARIAS. 2009. Aceite de sacha inchi NTP 151.400 Requisitos. (Peru).

14. CORZZINI, S.C.S.; BARROS, H.D.F.Q.; GRIMALDI, R.; CABRAL, F.A. 2017. Extraction of edible avocado oil using supercritical $\mathrm{CO}_{2}$ and $\mathrm{CO}_{2}$ / ethanol mixture as solvents. J. Food Engineering. 194:40-45.

15. NMX-F-052-SCFI-2008. 2008. Aceites y grasas -aceite de aguacate- especificaciones Normas mexicanas. Dirección generalde normas. México. 10p.
16. FANALI, C.; DUGO, L.; CACCIOLA, F.; BECCARIA, M.; GRASSO, S.; DACHÀ, M.; DUGO, P. 2011. Chemical characterization of Sacha inchi (Plukenetia volubilis L.) oil. J. Agric. Food Chem. 59:13043-13049.

17. INSTITUTO COLOMBIANO DE NORMAS TÉCNICAS Y CERTIFICACIÓN -ICONTEC-. 2002. Grasas de uso en mesa y cocina. Sexta actualización (NTC241). (Colombia).

18. INSTITUTO COLOMBIANO DE NORMAS TÉCNICAS Y CERTIFICACIÓN -ICONTEC-. 2009. Grasas Y Aceites Comestibles Vegetales y Animales (NTC 199). (Colombia).

19. KOUZOUNIS, D.; LAZARIDOU, A.; KATSANIDIS, E. 2017. Partial replacement of animal fat by oleogels structured with monoglycerides and phytosterols in frankfurter sausages. Meat Science, 130:38-46.

20. MORAN-VALERO, M.I.; VÍCTOR. M.; PINZONES RUIZ-HENESTROSA, V.M.; PILOSOF, A.M.R. 2017. Synergistic performance of lecithin and glycerol monostearate in oil / water emulsions. Colloids and Surfaces B: Biointerfaces. 151:68-75.

21. MOYANO, M.J.; MELÉNDEZ-MARTINEZ, J.; ALBA, J.; FRANCISCO, HEREDIA. J. 2008. A comprehensive study on the colour of virgin olive oils and its relationship with their chlorophylls and carotenoids indexes ( II ): CIELUV and CIELAB uniform colour spaces. Food Res. International. 41:513-521.

22. OPS/OMS. 2016. Factores De Riesgo De Las Enfermedades No Transmisibles En La Región De Las Américas: Consideraciones para fortalecer la capacidad regulatoria. Departamento de Enfermedades No Transmisibles y Salud Mental. (Washington, D.C). (1):14-15.

23. PALLA, C.; GIACOMOZZI, A.; GENOVESE, D.B.; CARRÍN, M.E. 2017. Multi - objective optimization of high oleic sun fl ower oil and monoglycerides oleogels : Searching for rheological and textural properties similar to margarine. Food Structure.12:1-14.

24. PARODI, P.W. 2009. Has the association between saturated fatty acids, serum cholesterol and coronary heart disease been over emphasized ? International Dairy J. 19(6-7):345-361.

25. PAUCAR-MENACHO, L.M.; SALVADOR-REYES, R.; GUILLÉN-SÁNCHEZ, J.; CAPA-ROBLE, J.; MORENO-ROJO, C. 2015. Estudio comparativo de las 
características físico-químicas del aceite de sacha inchi (Plukenetia volubilis L.), aceite de oliva (Olea europaea ) y aceite crudo de pescado. Scientia Agropecuaria. 6(4):279-290.

26. PEREZ-MONTERROZA, E.J.; MARQUEZ-CARDOZO, C.J.; CIRO-VELASQUEZ, H.J. 2014. Rheological behavior of avocado (Persea americana Mill , cv . Hass ) oleogels considering the combined effect of structuring agents. LWT - Food Science and Technology: 59:673-679.

27. PERNETTI, M.; VAN MALSSEN, K.; KALNIL, D.; FLÖTER, E.; BOT, ARJEN. 2007. Structuring edible oil with lecithin and sorbitan tri-stearate. Food Hydrocolloids. 21(5-6):855-861.

28. RINCÓN, S.M.; MARTíNEZ, D.M. 2009. Análisis de las propiedades del aceite de palma en el desarrollo de su industria. Palmas. 30(2):11-24.

29. SINGH, V.K.; PANDEY, P.M.; AGARWAL, T.; KUMAR, D.; BANERJEE, I.; ANIS, A.; PAL, K. 2016 . Development of soy lecithin based novel self-assembled emulsion hydrogels. J. Mechanical Behavior of Biomedical Materials. 55:250-263.

30. VARGAS-ORTIZ, M.; SERVENT, A.; SALGADO, C.M.; PALLET, D. 2017. Stability of the lipid fraction of avocado puree obtained by $\mathrm{fl}$ ash vacuum-expansion process. Innovative Food Science and Emerging Technologies. 41:109-116.
31. VICENTE, J.; GERALDO, M.; CARVALHO, D.; GARCIA, R.E.E. 2015. Fatty acids profile of Sacha Inchi oil and blends by 1 H NMR and GC - FID. Food Chemistry.181:215-221.

32. VIVES, C.J. 2004 . Comportamiento de una mezcla de aceite de soya y oleína de palma en fritura de papas a la francesa. Palmas. 25(1):308-311.

33. WANG, F.C.; MARANGONI, A.G. 2016. Microstructural basis for water release from glycerol monostearate structured emulsions upon transformation from the a -gel to the coagel phase. Food Structure. 7:1-5.

33. ZULETA SUAREZ, E.C.; RIOS, L.A.; BENJUMEA, P.N. 2012. Oxidative stability and cold flow behavior of palm, sacha-inchi, jatropha and castor oil biodiesel blends. Fuel Processing Technology. 102:96-101.

34. ZÚNIIIGA, L.A.; CIRO, H.J.; OSORIO, J. 2007. Estudio de la dureza del queso edam por medio de análisisanálisisde perfil de texturade perfil de textura y penetrometria por esferapenetrometria por esferapenetrometria por esfera. Rev. Fac. Nal. Agr.Medellín. 60(1):3797-3811.

Recibido: Octubre 8 de 2017

Aceptado: Abril 26 de 2018

Cómo citar:

Álvarez-H., M.E.; Ciro-V., H.J.; Arango-T., J.C. 2018. Caracterización fisicoquímica de oleogeles de aceite de aguacate (Persea americana) y sacha inchi (Plukenetia volubilis). Rev. U.D.C.A Act. \& Div. Cient. 21(1): 89-97. 